ISSN 0103-5150

Fisioter. Mov., Curitiba, v. 25, n. 2, p. 311-316, abr./jun. 2012 Licenciado sob uma Licença Creative Commons

\title{
Análise do equilíbrio estático em crianças com paralisia cerebral do tipo diparesia espástica com e sem o uso de órteses
}

\author{
Analysis of static balance in children with cerebral palsy \\ spastic diparetic type with and without the use of orthoses
}

\author{
Aryane Helena Roque ${ }^{[a]}$, Mariana Gonçalves Kanashiro ${ }^{[b]}$, Soraia Kazon ${ }^{[c]}$, \\ Luanda André Collange Grecco $^{[\mathrm{d}]}$, Afonso Shiguemi Inoue Salgado ${ }^{[\mathrm{e}]}$, Cláudia Santos de Oliveira ${ }^{[\mathrm{f}]}$ \\ [a] Fisioterapeuta graduada pela Universidade Nove de Julho (UNINOVE), São Paulo, SP - Brasil, e-mail: \\ aryrock87@gmail.com \\ [b] Fisioterapeuta e Mestranda em Ciências da Reabilitação na Universidade Nove de Julho (UNINOVE), São Paulo, SP - Brasil, \\ e-mail: mariana.kanashiro@yahoo.com.br \\ [c] Mestre em Ciências da Reabilitação pela Universidade Nove de Julho (UNINOVE), São Paulo, SP - Brasil, e-mail: \\ skazon@hotmail.com \\ [d] Fisioterapeuta e Mestranda em Ciências da Reabilitação na Universidade Nove de Julho (UNINOVE), São Paulo, SP - Brasil, \\ e-mail: luandacollange@terra.com.br \\ [e] Fisioterapeuta, Mestre pela Faculdade de Medicina da Universidade Estadual Paulista Júlio de Mesquita Filho (UNESP), \\ Doutorando em Engenharia Biomédica na Unicastelo, São Paulo - SP, Brasil, e-mail: afonsosisalgado@yahoo.com.br \\ [f] Docente do Programa de Pós-Graduação de Mestrado em Ciências da Reabilitação na Universidade Nove de Julho \\ (UNINOVE), São Paulo, SP - Brasil, e-mail: csantos@uninove.br
}

\section{Resumo}

Introdução: Crianças com paralisia cerebral (PC) do tipo diparesia espástica são frequentes usuárias de órteses para membros inferiores, com a finalidade de proporcionar melhora funcional da postura ortostática e durante a marcha. Objetivo: Analisar o equilíbrio estático de crianças diparéticas espasticas com PC, com e sem uso de órteses, por meio de parâmetros estabilométricos. Materiais e métodos: A amostra foi composta por 15 indivíduos com idades entre 4 e 14 anos (médias de 8,33 $\pm 2,74$ ), sete do sexo masculino e oito do sexo feminino, divididos em dois grupos: o grupo controle (GC) com seis participantes e o grupo estudado (GE) com nove crianças com PC diparesia espástica. Para a análise do equilíbrio estático utilizou-se uma plataforma de pressão (Medicapteurs Fusyo) e a coleta foi realizada com a criança em pé, em duas condições: com órteses e sem órteses. Resultados: 0 GE sem órtese apresentou maiores oscilações, estatisticamente 
significantes, no sentido médio-lateral e na área total de oscilação quando comparado ao desempenho do GC. Conclusão: Os resultados apresentados sugerem que a utilização de órteses para membros inferiores possa influenciar no equilíbrio estático, diminuindo a oscilação durante a postura ortostática estática.

Palavras-chave: Criança. Paralisia cerebral. Equilíbrio postural.

\begin{abstract}
Introduction: Children with spastic diparetic cerebral palsy (CP) are frequent users of orthoses for lower limbs, in order to provide functional improvement of posture during walking and standing. Objectives: To analyze the static balance of spastic diparetic children with CP, with and without the use of orthoses, through stabilometric parameters. Materials and methods: The sample comprised 15 individuals aged between 4 and 14 years old ( $8.33 \pm 2.74)$, seven males and eight females, divided into two groups: the control group (CG), with six participants, and the study group (SG), with nine spastic diparetic children with CP. For static balance analysis it was used a pressure platform (Medicapteurs Fusyo) and the collection was performed with the child standing on two conditions: with orthoses and without orthosis. Results: The EG with orthesis showed higher oscilation, statistically significant, mediolateral and the total area of oscillation compared to the GC. Conclusion: The results suggest that the use of orthoses for lower limbs can influence the static balance, reducing the oscillation during static standing position.
\end{abstract}

Keywords: Child. Cerebral palsy. Postural balance.

\section{Introdução}

Paralisia cerebral (PC) refere-se às desordens do desenvolvimento motor, advindas da lesão cerebral primária, de caráter permamente e mutável, que ocasionam alterações musculoesqueléticas secundárias e limitações nas atividades (1). Sabe-se que a principal alteração presente nas crianças com PC é o comprometimento motor, que acarreta várias modificações decorrentes da encefalopatia, com consequentes alterações na biomecânica corporal (2-9).

A diparesia espástica se caracteriza pelo acometimento mais intenso de membros inferiores, predominante na musculatura extensora e adutora (10). Os membros inferiores geralmente apresentam aumento da adução e rotação interna dos quadris, excessiva flexão dos joelhos associada ao valgismo e equinovaro $(10,11)$. Essas alterações biomecânicas, secundárias à espasticidade e à fraqueza muscular, podem resultar no comprometimento do equilíbrio estático e dinâmico $(12,13)$.

Deficits no controle postural têm sido identificados como a maior limitação no desenvolvimento motor de crianças com PC (14). 0 controle postural é um processo complexo que depende da integração da visão, da sensação vestibular e periférica, dos comandos centrais e respostas neuromusculares, particularmente da força muscular e do tempo de reação (15). A estabilidade postural é definida como a habilidade de manter e controlar o centro de massa do corpo dentro da base de suporte, a fim de prevenir quedas e dominar os movimentos desejados (16). Segundo Bigongiari et al. (17), as oscilações ocorrem em virtude da dificuldade de manter os segmentos do corpo alinhados em uma pequena base, os pés.

Atualmente, existem diversos testes para a mensuração do equilíbrio (18). A plataforma estabilométrica é um método fácil e eficaz, que oferece informações detalhadas do equilíbrio estático. Consiste em uma técnica de avaliação que permite identificar as oscilações posturais nas direções ântero-posterior (AP) e médio-lateral (ML) do centro de pressão (COP) $(18,19)$.

As órteses de posicionamento são frequentemente utlizadas para auxiliar no tratamento de crianças com PC. O suporte mecânico oferecido pela órtese suropodálica, por exemplo, visa minimizar ou corrigir o padrão equino, proporcionando melhora no padrão de marcha $(13,20)$, com menor gasto energético e melhor qualidade de vida $(21,22)$. Elas são indicadas para a sustentação de um segmento do corpo ou para a inibição de movimentos involuntários, tendo como objetivos aumentar a função, previnir deformidades e contraturas, manter as extremidades em posição 
funcional, auxiliar a função da musculatura fraca e facilitar o controle motor seletivo $(23,24)$.

O objetivo do presente estudo foi analisar o equilíbrio estático de crianças PC diparéticas, com e sem o uso de órteses suropodálicas, comparando as oscilações do COP, nos sentidos AP e ML e a área total de oscilação (ATO), entre crianças saudáveis e com PC diparéticas espásticas, com e sem o uso de órteses.

\section{Materiais e métodos}

O presente estudo, de caráter observacional, transversal e controlado, foi aprovado pelo Comitê de Ética e Pesquisa da Universidade Nove de Julho, sob o protocolo n. $134.237 / 2007$, e realizado em conformidade com a Resolução n. 196/96. Os responsáveis legais concordaram com a participação das crianças no estudo por meio da assinatura do Termo de Consentimento Livre e Esclarecido.

As crianças foram triadas na clínica OrtofisioVale, São José dos Campos, e na clínica escola da Universidade Nove de Julho. A amostra foi composta por 15 indivíduos com idades entre 4 e 14 anos $(8,3 \pm 2,7)$, de ambos os sexos. Os participantes foram divididos em dois grupos: grupo controle (GC), composto por seis crianças com desenvolvimento motor típico, sendo três do sexo masculino; e grupo estudado (GE), composto por nove crianças, sendo cinco do sexo masculino, com PC diparética espástica.

Foram incluídos no GE indivíduos com diagnóstico de PC capazes de permanecer na postura ortostática sem apoio e com grau de colaboração compatível com a realização dos procedimentos. O GS foi composto por indivíduos saudáveis que não apresentavam distúrbios neurológicos e/ou ortopédicos. Por outro lado, foram excluídos indivíduos com deficiência intelectual severa, deficit visual sem correção e distúrbios vestibulares.

A avaliação foi realizada em um único dia. Inicialmente, as características antropométricas (estatura e massa corporal) de cada criança foram mensuradas. Os participantes do GC realizaram as avaliações descalços, e os do GE, com e sem órteses suropodálicas fixas, confeccionadas especificamente para cada criança, previamente ao estudo.

0 equilíbrio estático foi avaliado por uma plataforma de pressão, com 2.300 sensores resistidos, da marca Medicapteurs, modelo fusyo. Esta permite uma análise estabilométrica por meio do registro dos deslocamentos COP, nos sentidos médio-lateral e ântero-posterior, e tempo de contato do pé com a superfície da plataforma. A frequência de aquisição foi de $40 \mathrm{~Hz}$. Para a avaliação, a criança foi orientada a permanecer em posição ortostática sobre a plataforma, descalça, com os braços ao longo do corpo, olhar fixo em um ponto marcado a um metro de distância na altura do ponto glabelar de cada criança, com base irrestrita dos pés e alinhamento dos calcanhares. Os dados foram registrados e interpretados pelo software Fusyo Analysis ${ }^{\circledR}$.

Os dados foram organizados e tabulados utilizando-se o programa SPSS (Statistical Package for the Social Sciences), versão 19.0. Os resultados estão expressos em média e desvio padrão. Os dados foram submetidos a uma análise de distribuição da frequência pelo teste Shapiro-Wilk, e a normalidade foi determinada a partir do teste paramétrico. $\mathrm{Na}$ análise intragrupo das crianças com PC, nas condições com e sem órteses foi utilizado o teste t dependente. A análise intergrupos (GE sem e com órtese e GC) foi utilizada ANOVA com post-hoc de Bonferroni. 0 nível de significância adotado foi de $\mathrm{p}<0,05$.

\section{Resultados}

A Tabela 1 apresenta os dados antropométricos da amostra estudada, de acordo com os grupos estudados, com relação à idade, massa corporal e estatura, expressas em: média \pm desvio padrão.

Os valores de deslocamento do COP (centro de pressão) no eixo médio-lateral (X) e ântero-posterior (Y) foram definidos pelo protocolo de Winter (19), onde:

Delta $\mathbf{X}(\Delta \mathbf{X})$ = máxima variação do COP na direção $X$ na série temporal $(\mathrm{em} \mathrm{mm})=$ máximo COPX - mínimo COPX

Tabela 1 - Características antropométricas dos participantes

\begin{tabular}{lcc}
\hline & $\begin{array}{c}\text { Grupo controle } \\
\text { (6 participantes) }\end{array}$ & $\begin{array}{c}\text { Grupo estudado } \\
\text { (9 participantes) }\end{array}$ \\
\hline Idade (anos) & $8,0 \pm 2,5$ & $8,5 \pm 3,0$ \\
Massa corporal (kg) & $33,8 \pm 13,6$ & $28,5 \pm 12,5$ \\
Estatura (cm) & $129 \pm 0,1$ & $124 \pm 0,1$ \\
\hline
\end{tabular}

Fonte: Dados da pesquisa. 
Delta $\mathbf{Y}(\Delta \mathbf{Y})$ = máxima variação do COP na direção $Y$ na série temporal $(\mathrm{em} \mathrm{mm})=$ máximo COPY - mínimo COPY

A área de deslocamento total do COP foi definida como:

\section{Área de deslocamento $=(\Delta \mathrm{X}) *(\Delta \mathrm{Y})\left(\mathrm{mm}^{2}\right)$}

A Tabela 2 apresenta os valores médios \pm desvio padrão das oscilações do COP, nos grupos estudados (GE sem e com órtese e GC).

Na análise comparativa entre os valores médios da área total de oscilação do COP foi observada diferença estatisticamente significante nas crianças com PC, com e sem o uso da órtese suropodálica fixa $(p=0,007)$. Na análise intergrupos, houve uma diferença estatística entre o GE sem órtese e o GC (p = 0,001) (Figura 1).

Não houve diferença estatística na oscilação no sentido ântero-posterior, na análise do GE sem e com

Tabela 2 - Oscilação do COP do GE sem e com órtese e do GC (média \pm desvio padrão)

\begin{tabular}{lccr}
\hline & \multicolumn{2}{c}{ Grupo estudado } & \multicolumn{1}{c}{$\begin{array}{c}\text { Grupo } \\
\text { Controle }\end{array}$} \\
\cline { 2 - 3 } & Sem órtese & Com órtese & \multicolumn{1}{c}{ Conn } \\
\hline Oscilação ML & $17,8 \pm 4,7$ & $10,4 \pm 7,6$ & $6,3 \pm 1,2$ \\
Oscilação AP & $34,5 \pm 25,7$ & $21,6 \pm 15,5$ & $10,5 \pm 4,5$ \\
Área total de & $3,3 \pm 0,5$ & $2,0 \pm 1,2$ & $1,5 \pm 0,4$ \\
oscilação & & & \\
\hline
\end{tabular}

Legenda: $\mathrm{ML}=$ médio-lateral e AP = ântero-posterior. Fonte: Dados da pesquisa.

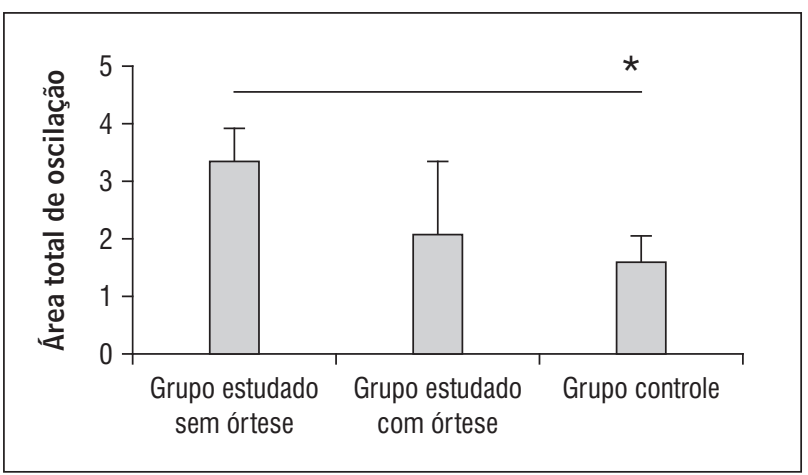

Figura 1 - Variação da área total de oscilação do COP do GE (com e sem órtese) e GC

Legenda: ${ }^{*}(p<0,01)$.

Fonte: Dados da pesquisa. órtese ( $p=0,77$ ) e na análise intergrupos (GE sem e com órtese e GC) $(p=0,20)$. No sentido médio-lateral observou-se diferença estatística na análise do GE sem e com órtese $(p=0,025)$ e na análise intergrupos na situação sem órtese do GE e o GC (p = 0,002) (Figura 2).

\section{Discussão}

As órteses suropodálicas são frequentemente prescritas para corrigir o desalinhamento musculoesquelético em crianças com diparesia espástica (13) e seus benefícios na marcha já foram bem descritos na literatura, porém pouco se sabe sobre os efeitos no controle postural estático em crianças com PC (16).

Segundo Näslund et al. (25), crianças com PC espástica que fazem uso de órtese suropodálica apresentam uma melhora do equilíbrio durante a marcha. Cury et al. (26) descrevem que o uso da órtese promove um melhor desempenho durante as tarefas motoras diárias e maior estabilidade durante a marcha de crianças com PC. No presente estudo, observou-se que as crianças com PC apresentaram menor oscilação da área total do COP com a utilização da órtese, demonstrando os benefícios da utilização desta, não apenas no equilíbrio funcional, mas no equilíbrio estático, ainda pouco estudado.

Segundo Nobre et al. (27), em crianças saudáveis, com as estratégias de equilíbrio íntegras, o ortostatismo é mantido sem a necessidade de adaptações das estratégias motoras globais. No entanto, o mesmo não acontece com as crianças com PC, pois não apresentam integridade das estratégias, incluindo a reação de tornozelo. Os resultados do presente estudo

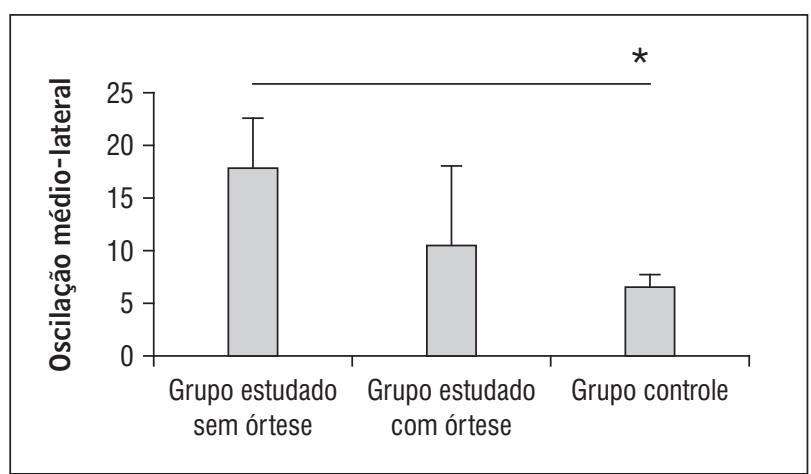

Figura 2 - Variação da oscilação do COP, no sentido médio-lateral entre os grupos (GE sem e com órtese e GC) Legenda: ${ }^{*}(p<0,01)$.

Fonte: Dados da pesquisa. 
contribuem para tais achados, uma vez que as crianças com PC sem a utilização da órtese suropodálica apresentaram maior oscilação quando comparadas às crianças saudáveis. Elas desenvolvem uma variedade de problemas no desenvolvimento neuropsicomotor, deficiência no sistema musculoesquelético e anormalidades no sistema sensorial, exigindo a alteração das estratégias motoras para a manutenção do equilíbrio. Donker et al. (28) encontraram a deteriorização do controle postural em crianças com PC quando comparadas a crianças com desenvolvimento neuronal normal. Burnet et al. (13) relataram que em crianças com espasticidade, durante as respostas de equilíbrio, ocorre a diminuição da área de contato do pé com o solo, levando a uma maior oscilação e dificuldade de manter o equilíbrio estático.

No presente estudo foi identificada diferença estatística no deslocamento total do COP no sentido ML entre o GE sem órtese e o GS. Já no estudo realizado por Rha et al. (18) foram analisadas as características do equilíbrio estático com e sem o uso de órteses articuladas tornozelo-pé em 21 crianças PC diparéticas. 0 resultado demonstrou que não houve diferença estatística quando comparadas as condições com e sem utilização de órtese em relação aos parâmetros de oscilação AP, ML e área do COP (16).

Segundo Allegretti et al. (29), o treinamento do equilíbrio, utilizando diferentes superfícies e materiais que estimulam as informações sensoriais, pode promover melhora da resposta do ajuste postural em pé. A prática é essencial para o aprendizado efetivo de tarefas complexas e também para a melhora da função motora (30). No presente estudo, as crianças com PC foram submetidas à avaliação estabilométrica em um único momento. Se as avaliações fossem realizadas em mais de um momento, possivelmente, os resultados seriam alterados, em virtude dos estímulos sensoriais e do fator de aprendizagem.

\section{Conclusão}

A órtese suropodálica fixa refletiu em menor oscilação do COP, no sentido médio-lateral e na área total de oscilação, ou seja, em melhor equilíbrio estático, na amostra estudada de crianças com PC do tipo diparesia espástica. Com a utilização desse tipo de órtese, as crianças com PC apresentaram oscilação semelhante às crianças sem comprometimento neurológico, saudáveis. Entretanto, estudos adicionais devem ser realizados com um número maior de participantes a fim de que os resultados sejam explorados de forma mais ampla.

\section{Agradecimentos}

Agradecemos ao suporte financeiro do Conselho Nacional de Desenvolvimento Científico e Tecnológico (CNPq), à Coordenação de Aperfeiçoamento de Pessoal de Nível Superior (CAPES) e à Fundação de Amparo à Pesquisa do Estado de São Paulo (FAPESP).

\section{Referências}

1. Rosenbaum P, Paneth N, Leviton A, Goldstein M, Bax $\mathrm{M}$, Damiano D, et al. A report: the definition and classification of cerebral palsy. April 2006. Dev Med Child Neurol Suppl. 2007;109:8-14.

2. Vasconcelos RLM, Moura TL, Campos TF, Lindquist ARR, Guerra RO. Avaliação do desempenho funcional de crianças com paralisia cerebral de acordo com níveis de comprometimento motor. Rev Bras Fisioter. 2009;13(5):390-7.

3. Palisano R, Rosenbaum P, Walter S, Russell D, Wood E, Galuppi B. Development and reliability of a system to classify gross motor function in children with cerebral palsy. Dev Med Child Neurol. 1997;39(4):214-23.

4. Kav i A, Vodušek D. A historical perspective on cerebral palsy as a concept and a diagnosis. Eur J Neurol. 2005;12(8):582-7.

5. Dzienkowski RC, Smith KK, Dillow KA, Yucha CB. Cerebral palsy: a comprehensive review. Nurse Pract. 1996;21(2):45-8, 51-4, 57-9.

6. Knox V, Evans AL. Evaluation of the functional effects of a course of Bobath therapy in children with cerebral palsy: a preliminary study. Dev Med Child Neurol. 2002;44(7):447-60.

7. Awaad Y, Tayem H, Munoz S, Ham S, Michon AM, Awaad R. Functional assessment following intrathecal baclofen therapy in children with spastic cerebral palsy. J Child Neurol. 2003;18(1):26-34.

8. Schwartzman JS. Paralisia cerebral. Arq Bras Paralisia Cerebral. 2004;1(1):4-17. 
9. Manoel EDJ, Oliveira J. Motor developmental status and task constraint in overarm throwing. J Human Mov Studies. 2000;39(6):359-78.

10. Rotta NT. Paralisia cerebral: novas perspectivas terapêuticas. J Pediat. 2002;78(Supl 1):S48-S54.

11. Hägglund G, Lauge-Pedersen $H$, Wagner P. Characteristics of children with hip displacement in cerebral palsy. BMC Musculoskelet Disor. 2007;8(1):101-16.

12. Ferrarezi KC, Guedes JERP. O uso de técnicas para auxiliar a flexibilidade e equilíbrio em adolescentes portadores de paralisia cerebral: o relato de três casos. Acta Scientiarum. 2008;2(22):625-9.

13. Burtner PA, Woollacott MH, Qualls C. Stance balance control with orthoses in a group of children with spastic cerebral palsy. Dev Med Child Neurol. 1999; 41(11):748-57.

14. Rose J, Wolff DR, Jones VK, Bloch DA, Oehlert JW, Gamble JG. Postural balance in children with cerebral palsy. Dev Med Child Neurol. 2002;44(1):58-63.

15. Overstall $P$. The use of balance training in elderly people with falls. Rev Clin Gerontology. 2003;13(2): 153-61.

16. Swanenburg J, De Bruin ED, Favero K, Uebelhart D, Mulder T. The reliability of postural balance measures in single and dual tasking in elderly fallers and nonfallers. BMC Musculoskelet Disord. 2008;9:162.

17. Bigongiari A, Corrêa JCF, Corrêa FI, Franco RC. Corporal oscilation during static biped posture in children with cerebral palsy. Electromyogr Clin Neurophysiol. 2007;47(3):131-6.

18. Rha D, Kim DJ, Park ES. Effect of hinged ankle-foot orthoses on standing balance control in children with bilateral spastic cerebral palsy. Yonsei Med J. 2010; 51(5):746-52.

19. Blaszczyk J, Orawiec R, Duda-K odowska D, Opala G. Assessment of postural instability in patients with Parkinson's disease. Exp Brain Res. 2007;183(1): 107-14.

20. Romkes J, Brunner R. Comparison of a dynamic and a hinged ankle-foot orthosis by gait analysis in patients with hemiplegic cerebral palsy. Gait Posture. 2002; 15(1):18-24.
21. Radtka SA, Skinner SR, Dixon DM, Johanson ME. A comparison of gait with solid, dynamic, and no ankle-foot orthoses in children with spastic cerebral palsy. Phys Ther. 1997;77(4):395-409.

22. Carlson WE, Vaughan CL, Damiano DL, Abel MF. Orthotic management of gait in spastic diplegia. Am J Phys Med Rehabil. 1997;76(3):219-25.

23. Winter DA. Human balance and posture control during standing and walking. Gait Posture. 1995;3(4): 193-214.

24. Mattacola CG, Dwyer MK, Miller AK, Uhl TL, McCrory JL, Malone TR. Effect of orthoses on postural stability in asymptomatic subjects with rearfoot malalignment during a 6-week acclimation period. Arch Phys Med Rehabil. 2007;88(5):653-60.

25. Naslund A, Sundelin G, Hirschfeld H. Reach performance and postural adjustments during standing in children with severe spastic diplegia using dynamic ankle-foot orthoses. J Rehabil Med. 2007;39(9):715-23.

26. Cury V, Mancini M, Melo A, Fonseca S, Sampaio R. Efeitos do uso de órtese na mobilidade funcional de crianças com paralisia cerebral. Rev Bras Fisioter. 2006;10(1):67-74.

27. Nobre A, Monteiro F, Golin M, Biasotto-Gonzalez D, Corrêa J, Oliveira C. Analysis of postural oscillation in children with cerebral palsy. Electromyogr Clin Neurophysiol. 2010;50(5):239-44.

28. Donker SF, Ledebt A, Roerdink M, Savelsbergh GJP, Beek PJ. Children with cerebral palsy exhibit greater and more regular postural sway than typically developing children. Exp Brain Res. 2008;184(3):363-70.

29. Allegretti KMG, Kanashiro MS, Monteiro VC, Borges HC, Fontes SV. Os efeitos do treino de equilíbrio em crianças com paralisia cerebral diparética espástica. Rev Neurocienc. 2007;15(2):108-13.

30. Walker C, Brouwer BJ, Culham EG. Use of visual feedback in retraining balance following acute stroke. Phys Ther. 2000;80(9):886-95.
Recebido: 02/06/2011

Received: 06//02/2011

Aprovado: 25/09/2011

Approved: 09/25/2011 